\title{
CFOs werden mit Cloud und KI zur Dateninstanz
}

Schon immer verfügte die Finanzfunktion über die meisten Daten. Jetzt steht ihr eine Fülle neuer Daten zur Verfügung. Mit einem cloudbasierten Datenökosystem schafft sie die Voraussetzungen für den Einsatz von Künstlicher Intelligenz und damit neue Möglichkeiten für die Unternehmenssteuerung und eine zukunftssicherere Ausrichtung von Unternehmen.

Andreas Braun 
Nie schien der Einsatz von Künstlicher Intelligenz (KI) in Finanzabteilungen relevanter als gegenwärtig: Hätte KI nicht Anomalien in den Daten und Transaktionen bei Wirecard finden müssen? Liefern nicht gerade moderne Datenplattformen die Möglichkeit, einfacher als je zuvor schnell verschiedene Szenarien in der COVID-19-Krise durchzuspielen? Tatsächlich kann auch KI keine pandemischen Ereignisse vorhersagen. Sie kann aber historische Unternehmensinformationen und externe Informationen als TrainingsDaten nutzen, um die Auswirkungen von komplexen Veränderungen zu prognostizieren und verschiedene Szenarien durchzuspielen. Genau dies macht KI für das Controlling und die Unternehmenssteuerung relevant.

\section{Die Finanzfunktion als Datendrehscheibe}

Daten sind der notwendige Rohstoff für Digitalisierung sowie für den Einsatz neuer Technologien wie KI. Kann die Finanzfunktion ein Datenökosystem, verstanden als eine gemeinsame Datenschicht, die alle für ein Unternehmen relevanten internen und externen Daten einbezieht, etablieren, kann sie zur strategischen Datendrehscheibe im Unternehmen werden. Solch ein Datenökosystem liefert zu jedem Zeitpunkt eine gemeinsame Sicht auf Finanzdaten und operative Betriebsdaten, einen sogenannten „Single Point of Truth“. Die Zusammenführung der Daten in einer Dateninstanz, auf die man sich universell verlassen kann und die unternehmensweit verfügbar ist, wirkt zudem einer bisher weitverbreiteten Silo-Bildung entgegen.

Schon bisher konnten Finanzabteilungen mittels ERP-Systemen die aktuelle Unternehmenssituation zeitnah deskriptiv erfassen und waren in der Lage, lineare Prognoseszenarien zu erstellen. Mit zunehmenden Datenpunkten und -mengen können die Vorhersagen noch genauer werden, allerdings wächst auch die Herausforderung: Mit dem Zugriff auf und der Integration von immer mehr Datenquellen steigt die Komplexität und damit auch die Fehleranfälligkeit.

\section{Die Cloud als Enabler}

Um alle Daten in einer einzigen gemeinsamen Datenschicht zusammenzuführen, kommt der Cloud-Technologie eine wichtige Rolle als Enabler und Beschleuniger zu. Bis vor Kurzem war es technisch nicht möglich, einen einzigen operativen und dispositiven Datensatz zu haben. Grund dafür war, dass transaktionale operative Systeme andere Anforderungen an den Datenzugriff stellen als skalierbare Systeme, wie sie üblicherweise für Big Data, KI und letztlich auch Business-Intelligence-Lösungen verwendet werden. Der Vorteil der Zusammenführung in einem Datenökosystem ist, dass komplexe und alte Applikationen weitestgehend unberührt bleiben können. Dieses Vorgehen ist kosteneffizienter und verbessert die Analysegeschwindigkeit um ein Vielfaches. Zudem wird eine spätere Modernisierung der Systeme einfacher. Der deutlichste Vorteil liegt darin, dass es für alle Beteiligten zu jedem Zeitpunkt nur genau eine „wahre“ Sicht auf die Daten gibt. Komplexität, Kosten und die IT-Architektur werden drastisch vereinfacht, wäh-

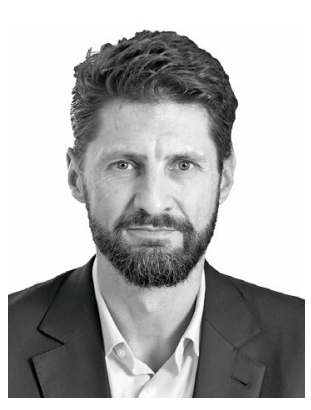

\section{Dr. Andreas Braun}

ist Managing Director bei Accenture und verantwortet das Applied-Intelligence- und Data-Group-Geschäft in Europa.

E-Mail: andreas.braun@accenture.com 


\section{Die Entkoppelung der Daten} von komplexen (Finanz-) Applikationen trägt dazu bei, Silos zu überwinden. rend die Nutzung von veralteten oder inkorrekten Daten für Analysen und Berichte ausgeschlossen wird.

Dabei ist es wichtig, die Cloud nicht einfach als ein anderes Rechenzentrum, betrieben von Dritten, zu verstehen. Die Cloud ist ein neues ITParadigma. Sie macht eine Unmenge von Diensten möglich, insbesondere in den Bereichen Daten, Maschinelles Lernen und KI. Ihr Wert liegt in der intelligenten Nutzung dieser „Mehrwert“-Dienste zur Beschleunigung und Vereinfachung. Das Cloud-Paradigma macht skalierbare, hybride Datenplattformen möglich, auf denen die unterschiedlichen Frontends von Unternehmens-Software, wie beispielsweise Kundenbeziehungs-Management (CRM) oder Finanz-, Steuer- und Abrechnungs-Software, auf Datenebene zusammenkommen. Das Verwenden einer gemeinsamen Datenschicht liefert nicht nur zeitnahe und aktuellere Vorhersagen im Vergleich zu ERP-Systemen. Durch die Integration aller internen sowie externen Daten sind nun auch neue, prädiktive Analyse-Methoden auf Basis von Maschinellem Lernen möglich, ohne den komplexen, fehlerbehafteten, langsamen und teuren Umweg über Kopien in sogenannten Datalakes oder Data Warehouses zu gehen.

\section{„Der Vorteil der Zusammenführung in einem Da- tenökosystem ist, dass komplexe und alte Applika- tionen weitestgehend unberührt bleiben können."}

Ausgehend von diesen neuen Möglichkeiten kommt gerade aufCFOs und

\section{Zusammenfassung}

- Die Finanzfunktion kann mittels neuer Technologien wie Cloud und KI zur Datendrehscheibe im Unternehmen werden und wesentliche datenbasierte Impulse für die Unternehmenssteuerung erarbeiten.

- Voraussetzung ist die Schaffung eines Datenökosystems, einer gemeinsamen Datenschicht unter Einbeziehung aller relevanten internen und externen Daten.

- Mit dieser Grundlage ergeben sich neue Möglichkeiten, wie schnelle Prognosen auf Basis von abgeänderten Rahmenbedingungen oder die Warnung vor Trend-Änderungen, zum Beispiel im Hinblick auf Märkte, Produkte oder Wettbewerber.
Finanzverantwortliche damit eine bedeutende neue Rolle zu. Sie sind seit jeher die zentrale Schnittstelle im Unternehmen, um Prognosen für die Entwicklung ihrer Unternehmen vorzunehmen - und damit sind sie natürlich auch im Besitz der wesentlichsten Datenbestände. Mit der Möglichkeit, Daten von komplexen (Finanz-)Applikationen zu entkoppeln und Silos (noch immer gibt es in vielen Unternehmen unterschiedliche Datenbanken in den verschiedenen Abteilungen oder Business Units) zu überwinden, werden CFOs zur Dateninstanz im Unternehmen.

Mit dem Einzug und der zunehmenden Reife von Big Data, Cloud und KI bietet sich mittlerweile ein neuer Ansatz für zukunftsgerichtete genaue Prognosen aus der Kombination der Basistechnologien. Unter anderem sind vor allem Qualität und Menge der Daten entscheidend. Die in vielen Unternehmen vorhandenen Datensilos verhindern einen effektiveren Einsatz aber zunehmend. Laut einer im Auftrag von Rubrik erstellten IDC-Studie (IDC 2020) investierten Unternehmen weltweit 900 Milliarden US-Dollar in die Digitalisierung. Davon waren laut Studie 70 Prozent „reine Geldverschwendung" und haben die Unternehmen nicht weitergebracht, weil Datensilos nicht beseitigt, sondern neue Datensilos geschaffen wurden. Die Zusammenführung der Daten in einer Dateninstanz ermöglicht eine einheitliche Sicht, den „Single Point of Truth“. Es geht um die Schaffung eines allgemein- 
gültigen, unternehmensweit verfügbaren Datenbestands, auf den man sich universell verlassen kann.

\section{Künstliche Intelligenz}

KI beschreibt Technologien, die Maschinen befähigen, Dinge zu tun, die man normalerweise menschlichem Intellekt zuschreiben würde. KI unterscheidet sich damit deutlich von Computer-Programmen, wie wir sie vormals kannten. Während Software und Algorithmen von Menschen programmiert werden, die Intelligenz der Software-Entwickler also in ein Computer-Programm kodifiziert wurde, ist KI lernfähig. KI kann auf Basis von Daten trainiert werden, eigenständige Entscheidungen zu treffen (Maschinelles Lernen). Da sich Daten dynamisch ändern, verändert die KI ihr Verhalten und lernt kontinuierlich weiter. Die Daten können und sollen dabei durchaus sehr komplex und umfangreich sein, exaktes analytisches Verständnis über Ursache-Wirkungs-Zusammenhänge aber ist nicht notwendig - anders als bei der Programmierung. Das heißt konkret, dass KI Muster in umfangreichen historischen Daten „lernen“ kann, die in komplexen Zusammenhängen auf ein Ergebnis beziehungsweise Ereignis hindeuten und dieses vorhersagen. Die erkannten Muster sind in der Regel sehr komplex und für Menschen auf Anhieb nicht unbedingt nachvollziehbar, da sie interne und externe Ereignisse sowie Trigger zusammenfügen - Stichwort „Blackbox“. Das können komplexe Querbeziehungen von politischen, wirtschaftlichen und ökologischen Ereignissen sein, die sich gegenseitig beeinflussen und frühzeitig auf ein Resultat hindeuten. Wichtig ist, dass die Daten so breit gefächert sind, dass wesentliche Ereignisse enthalten sind.

\section{„Es geht um die Schaffung eines allgemeingültigen, unternehmensweit verfügbaren Datenbestands, auf den man sich universell verlassen kann."}

Die Vorteile des Einsatzes von KI stehen und fallen mit der Qualität der dem Lernen zugrunde liegenden Daten. Je niedriger deren AbstraktionsLevel, desto besser das Ergebnis. Die Ergebnisse sind in der Regel als Wahrscheinlichkeiten zu betrachten. Akzeptiert man das, so ergeben sich diverse Nutzungsmöglichkeiten für CFOs: Der Finanzbereich verfügt über komplexe und umfassende Daten, obendrein üblicherweise mit langer Historie. Nimmt er externe Daten, zum Beispiel verwandter Sektoren, Länder oder Nachrichten hinzu, so kann er fundiertere oder sogar völlig neue Aussagen treffen.

\section{Beispielanwendungen}

Die Nutzungsmöglichkeiten von Cloud-Datenökosystemen und KI in der Finanzfunktion nehmen stetig zu. Zwei Beispiele zeigen Nutzungsmöglichkeiten im Rahmen der Prozessoptimierung und Szenario-Erstellung.

\section{Datenökosysteme vereinfachen die Datennutzung in Unterneh- men erheblich.}




\section{Cloudbasierte Datenökosysteme erleichtern den Einsatz von KI und damit von komplexen Simulationsmodellen.}

\section{Cloud-Datenökosysteme in der Finanzindustrie}

Ein globaler Konzern der Finanzwirtschaft nutzt cloudbasierte Datenökosysteme, um perspektivisch alle Daten in einer einzigen gemeinsamen Datenschicht zusammenzuführen. Ein Treiber hierfür ist der Einsatz von KI, der für das Unternehmen einen enormen Beitrag zur Prozessoptimierung vor allem auch regulatorischer Prozesse leisten kann. Auch bei der Definition und Umsetzung neuer Geschäftsmodelle - wie beispielsweise skalierbaren „As-aService“-Finanzprodukten - spielt KI die entscheidende Rolle. Ein weiterer Treiber war, dass das Datenökosystem zu jedem Zeitpunkt eine gemeinsame Sicht auf Finanzdaten und operative Betriebsdaten liefert. Das Problem, dass auf verschiedenen Wegen erzeugte Berichte nicht dieselben Ergebnisse liefern

\section{„CFOs sollten möglichst sofort KI in die Finanz- funktion integrieren."}

und dass damit der Einsatz einer KI ausgehebelt wird, wird somit gelöst. Die eingesetzte regulatorische KI hat somit eine Gesamtsicht und kann aus Transaktionen des Betriebs ableiten, ob eine Finanztransaktion ein Betrugs- oder Geldwäschefall sein könnte - und die Transaktion gegebenenfalls automatisch aussetzen. Auch können tagtäglich stattfindende Verkäufe, Stornierungen, Wettbewerbssituationen oder Veränderungen in bestimmten Märkten sofort in Prognosen und Hochrechnungen überführt werden und Gegenmaßnahmen, wie Kundensicherung für ein bestimmtes Land/Produkt, modelliert werden - ebenfalls fast in Echtzeit und automatisch.

\section{KI für Szenarien zu veränderten Rahmenbedingungen}

Ein mittelgroßer Versicherer experimentiert aktuell mit der Nutzung virtualisierter Datenkopien, also einer Referenz seiner originalen Finanzdaten, mit der Möglichkeit, alternative Szenarien durchzuspielen, ohne das Original zu überschreiben und dennoch Änderungen stets in den Szenarien zu reflektieren. So können zum Beispiel bestimmte geschäftsrelevante Parameter für prädiktive Modellrechnungen entsprechend fiktiver Rahmenbedingungen modifiziert und automatisch neue Ergebniswerte, wie Abschlüsse, auf Basis der neuen Annahmen berechnet werden.

Auf Basis der gelernten Daten, über zum Beispiel verschiedene Abschlussjahre beziehungsweise unter Berücksichtigung verschiedener Regionen und Länder, kann ein Lernalgorithmus in Sekunden ein neues Szenario erstellen und somit sowohl die Auswirkungen von Trend-Änderungen als auch die Bandbreite möglicher Gegenmaßnahmen vorhersagen.

Ein Beispiel dazu: Die Rechtsprechung verändert sich, insbesondere bei Schadensereignissen, und hat eine erhebliche Auswirkung auf die zu zahlenden Schadenssummen und damit den Abschluss, allerdings auch auf Prämien in Folgejahren und damit auf Kündigungen. Maschinelles Lernen kann diese Szenarien auf Basis gelernter Modelle auffüllen und nicht nur Prognosen, sondern auch mittels eines unüberwachten Lernalgorithmus Lösungs- 
wege aufzeigen. Der erhebliche Unterschied zu traditionellen Methoden ist, dass kein komplexes mathematisches Modell benötigt wird, sondern nur passende Daten. Diese können sogar synthetisch, zum Beispiel aus historischen Daten oder Referenzländern, erstellt werden.

\section{Schlussbetrachtung}

Der Finanzbereich kann der Nukleus für die datengetriebene Erneuerung eines Unternehmens werden, und der CFO und sein Team können zukünftig weitgehend die Unternehmensstrategie vorantreiben. Über die meisten Daten verfügte die Finanzfunktion schon immer. Doch jetzt stehen ihr auch neue Technologien zur Verfügung, um diese Daten zu nutzen.

Insbesondere die Erstellung von greifbaren Szenarien mithilfe von KI ist für die Entscheidungsfindung der Unternehmensführung relevant. Sie hat so die Möglichkeit, verschiedene Ausrichtungen vorab auf ihre Zukunftsfähigkeit zu testen. Neue Produkte lassen sich in verschiedenen Varianten und mit unterschiedlichen Verfügbarkeitsdaten in die Simulation integrieren, um so den bestmöglichen Weg zu finden, ein Unternehmen in eine erfolgreiche Zukunft zu führen. Die dem Verfahren zugrunde liegende Simulationsmethodik ist dabei von der Branche unabhängig.

CFOs sollten möglichst sofort KI in die Finanzfunktion integrieren. Der Finanzbereich ist aufgrund seiner Datenhistorie ein erstklassiger Startpunkt, denn: Alle neuen Methoden basieren auf Daten; diese müssen sukzessive zusammengetragen werden, am besten über konkrete Anwendungen. Die Schaffung einer gemeinsamen Datenplattform beziehungsweise Datenökosystems kann technologieseitig über die Cloud gelöst werden. Kollaborative Ansätze bieten sich bei der Umsetzung an. Auch Partnerschaften machen Sinn, denn es gibt bereits viele Best Practices sowie Anwendungsfall-Listen, sodass niemand alte Fehler wiederholen muss.

Zuletzt muss bedacht werden, dass Innovationen nur dann vorangetrieben und akzeptiert werden, wenn sich auch das Mindset verändert, also gleichzeitig organisatorische und kulturelle Veränderungen vorangetrieben werden. Diese aber brauchen Zeit.

\section{Literatur}

IDC - International Data Corporation (Hrsg.) (2020): The data-forward enterprise: how to maximize data leverage for better business outcomes. White Paper Study, Framingham.

\section{SpringerProfessional}

\section{Kl erleichtert die Nutzung von Szenarien im Rahmen der Entscheidungsfindung.}

Fink, V. (2020): Die Guten ins Töpfchen - Daten essen die Welt, in: Quick Guide

KI-Projekte - einfach machen, Wiesbaden, S. 71-84.

www.springerprofessional.de/link/17587998

Ludwig, T./Nagel, W. E./Yahyapour, R. (2020): Das Ökosystem der Datenwissen-

schaften, in: Informatik Spektrum, 42, S. 432-440.

www.springerprofessional.de/link/17384762 